\title{
Von der Wissenschaftssoziologie zur Soziologie wissenschaftlichen Wissens
}

\author{
JÖRG NIEWÖHNER
}

Robert K. Merton begründete in den 1930 und 40er Jahren die Wissenschaftssoziologie mit seinen Untersuchungen über die Entwicklung der Wissenschaft im England des 17. Jahrhunderts und ihren wechselseitigen Zusammenhang mit gesellschaftlichen Entwicklungen, speziell dem Puritanismus. Wissenschaft wird durch diesen Forschungsansatz als Institution bzw. als Organisation erstmals Untersuchungen durch die Sozialwissenschaften zugänglich. Aus diesem Verständnis von Wissenschaft heraus entwickelt Merton seine Imperative des wissenschaftichen Ethos: Universalität, Kommunismus, Uneigennützigkeit und organisierter Skeptizismus. Die Wissenschaftssoziologie der Nachkriegszeit wird vor allen Dingen von immer neuen Angriffen auf diesen Ethos und Vereinnahmungsversuchen der Wissenschaft durch politische und wirtschaftliche Interessen geprägt. Die Technokratiedebatte und der Positivismusstreit in Deutschland, und die Untersuchungen und Streitschriften für wissenschaftliche Unabhängigkeit eines Michael Polanyi oder Jerry Ravetz in der anglo-amerikanischen Soziologie, sind so immer Ausdruck einer grundlegenden und hart geführten Debatte über Erkenntnismöglichkeiten in konkreten sozialen Institutionen. Nicht gestellt wird die Frage nach den Auswirkungen von institutionellem Wandel auf Wissen selbst. Dies ist das entscheidende Forschungsdesiderat, dessen sich die Science and Technology Studies in den 1970er Jahren annehmen: sie überführen die Wissenschaftssoziologie und ihre Untersuchun- 
gen von Wissenschaft als Institution in eine Soziologie wissenschaftlichen Wissens und machen damit Wissen selbst zum Untersuchungsgegenstand sozialwissenschaftlicher Forschung.

\section{Weiterführende Literatur}

Gieryn, Thomas F. (1983): »Boundary-Work and the Demarcation of Science from Non-Science: Strains and Interests in Professional Ideologies of Scientists«. American Sociological Review 48(6), S. 781-795.

Der Soziologe Thomas Gieryn zeichnet in diesem Artikel im Stile eines Robert Merton die verschiedenen Strategien nach, die Wissenschaftler verwenden, um ihre eigene Arbeit als wissenschaftlich darzustellen und damit klar von technischen oder handwerklichen Tätigkeiten abzugrenzen. Ein wichtiger Teil der Arbeit von Forschenden wird damit zur "Grenzarbeit" ein Konzept, auf das in der Wissenschaftsforschung in verschiedenen Facetten immer wieder verwiesen wird.

Bourdieu, Pierre (1975): »The Specificity of the Scientific Field and the Social Conditions of the Progress of Reason. [Volkskunde] «. Social Science. Infrmation sur les sciences sociales XIV-(6), 19-47.

Ein Klassiker der Kulturwissenschaft: Bourdieu entwickelt in diesem Aufsatz seinen »Feld« Begriff und zeigt anschaulich, wie wissenschaftliche Arbeit und das Streben nach Plausibilisierung von Ideen immer auch ein Streben nach Autorität und Deutungshoheit darstellt. 


\section{Einleitung}

Die zentralen wissenschaftstheoretischen Positionen, die im vorangegangenen Kapitel kurz vorgestellt worden sind, beruhen mit Ausnahme von Ludwik Fleck auf theoretischen, historischen und normativen Überlegungen. Sie untersuchen den Erkenntnisprozess und damit auch den Charakter wissenschaftlichen Wissens entweder theoretisch als Ergebnis formallogischer oder rationaler Folgerungen, oder auf der Basis von historischen Fallstudien, d. h. vergangenen Entwicklungen in bestimmten Wissenschaftsfeldern, die aus Quellenanalysen und Sekundärliteratur erschlossen werden. Für Forschende in der Kulturanthropologie sind das relevante, aber nicht zwingend die zentralsten Zugänge. Wichtiger ist hier die Frage, wie Wissenschaft, bzw. der Prozess der Produktion wissenschaftlichen Wissens konkret von statten geht. Wie operiert Wissenschaft im Alltag und wie entstehen Erkenntnis und Wissen als Ergebnis von lokal, sozial und kulturell spezifischer Praxis? Diese Fragen nach Wissenspraxis sind wissenschaftstheoretisch nicht befriedigend zu beantworten, und doch wird bereits in den 1930er und 40er Jahren deutlich, dass diese Fragen nach den konkreten Bedingungen von Wissenschaft und Wissensproduktion für moderne Gesellschaften eine große Relevanz besitzen. Wissenschaftliches Wissen prägt zunehmend Alltage, Arbeitsprozesse und soziale Ordnung. Seine Entstehungskontexte besser zu verstehen, ist daher ein wichtiger Aspekt für das Verständnis gesellschaftlicher Ordnungsprozesse im Allgemeinen. ${ }^{1}$

Die Sozio-Logik von Wissenschaft avanciert also Mitte des 20. Jahrhunderts $\mathrm{zu}$ einem wichtigen Thema - vornehmlich als eine Frage der Soziologie. Die Anthropologie interessiert sich zu diesem Zeitpunkt zwar ebenfalls für die Zusammenhänge zwischen Erkenntnis, Wissen und gesellschaftlicher Ordnung, dies aber beinahe ausschließlich außerhalb von EuroAmerika, bzw. der westlichen Welt. Die Untersuchung nicht-westlicher

\footnotetext{
1 Gerade in dieser Hinsicht verschränken sich selbstverständlich Wissen und Technologie auf mannigfaltige Art und Weise. Fortschritt und Wachstum in westlichen Gesellschaften sind untrennbar mit Wissenszuwachs und technologischer Entwicklung verbunden. In diesem Kapitel liegt der Fokus jedoch nur auf den Anfängen der Wissenschaftssoziologie. Die verschiedenen Untersuchungen der Entwicklung und Rolle von Technologie für Gesellschaft finden sich im Kapitel SoZIALE KONSTRUKTION VON TECHNOLOGIE.
} 
Gruppen von Menschen als »Andere« der westlichen, modernen Rationalität, konstruiert deren Wissenspraktiken als defizitär und als Vorstufe, für die Magie und Zauber, Irrationalität oder primitives, d. h. noch nicht entwickeltes Wissen charakteristisch seien. (Warneken 2006, Kuper 1988, Kuper 2005 und RATIONALITÄT) Nach heutiger Sicht verraten uns diese Analysen zwar mindestens genauso viel über westliche Rationalität wie über das Wissen der »Primitiven«, weil in diesen Studien zumindest implizit westliche Rationalitätskonzepte als Maßstab herangezogen oder diese hinsichtlich ihres Universalitätsanspruches in Kontrastierung mit nichtwestlichen Handlungsmustern hinterfragt werden. Damals jedoch stand nur für wenige Forschende der Anthropologie die kulturkritische Überlegung im Vordergrund, dass die Untersuchungen des Fremden immer auch das Eigene mit beleuchten.

Die Untersuchung von Wissenschaft als organisiertem Prozess entwickelt sich demnach zu einem Forschungsfeld, das vor allem von der Soziologie, weniger von anderen Sozialwissenschaften oder der Philosophie dominiert wird. Es entsteht die Wissenschaftssoziologie. Sie erwächst in den 1930er und 1940er Jahren vornehmlich aus einer bereits etablierten Organisationssoziologie und untersucht Wissenschaft zunächst als eine Organisationsform von Handlung oder als eine Institution wie andere auch. Mit dieser soziologischen Perspektive - dies sei hier eingefügt, weil es bis heute eine Relevanz hat - geht allerdings ein Verlust an epistemologischen Fragestellungen einher. Die soziologische Untersuchung Wissen schaffender Institutionen vernachlässigt bis heute vielfach die Frage nach den epistemologischen Konsequenzen dieser institutionellen Strukturen. ${ }^{2}$ Was Ludwik Fleck gefordert hatte, nämlich eine vergleichende Epistemologie, die wissenschaftliche Praxis auf ihre Verschränkung mit Erkenntnisgewinn hin untersucht und dabei sowohl etwas über Wissen und Wissenschaft als auch über Gesellschaft verrät, gerät mehr oder minder in Vergessenheit und führt bis heute eher ein Nischendasein. Eine gewiss nicht intendierte Konsequenz dieses Zugriffs auf Wissenschaft als Institution ist eine Weiterführung der

2 Dies hat selbstverständlich weniger mit der Disziplin Soziologie an sich zu tun, als vielmehr mit dem häufig mit dieser Disziplin verbundenen quantifizierenden $\mathrm{Zu}$ griff. Der Beitrag zu Laborstudien in diesem Band (LABORSTUDIEN) zeigt, dass einige soziologische Studien gerade dort, wo sie auf qualitative und ethnographische Methoden setzen, wissenschaftliche Arbeit und epistemologische Konsequenz als verknüpft untersuchen. (Knorr-Cetina 1981) 
wahrgenommenen Sonderrolle oder Exzeptionalität von wissenschaftlichem Wissen im Vergleich zu anderen Wissensformen. Wissenschaftliches Wissen wird, wie in der Wissenschaftstheorie, nach wie vor weitgehend getrennt von gesellschaftlicher Entwicklung untersucht.

Im Gegensatz dazu hatte der US-Amerikanische Philosoph John Dewey bereits um die Jahrhundertwende zum 20. Jahrhundert dafür plädiert, wissenschaftliches und alltägliches Denken entlang eines Kontinuums zu konzeptionalisieren. (Dewey 2008: 86f.) Diese pragmatistische Position findet in zahlreichen Debatten der letzten zwanzig Jahre in der Wissenschaftsforschung (vgl. etwa Knorr-Cetina 1999) sowie der Anthropologie großen Widerhall. Auch hier wird zunehmend betont, dass Wissenschaft keineswegs vor- oder außerkulturell konzipiert werden dürfe. Wissenschaft, so das Argument der Anthropologie, sei eng mit gesellschaftlichem Wandel verwoben, als Teil von Kultur, nicht als außerhalb von ihr stehend oder ihr gar übergeordnet. (Vgl. Kontopodis et al. 2011) Wenn Wissenschaft in modernen Gesellschaften auch eine zentrale Rolle zukommt, so rechtfertigt dies keineswegs die Annahme eines prinzipiellen Unterschiedes zwischen verschiedenen Wissenspraxen.

\section{Die Anfänge der Wissenschaftssoziologie: ROBERT K. MERTON}

Die Soziologie der 1930er und 1940er Jahre ist stark strukturfunktionalistisch geprägt. Untersuchungen von Praxis spielen darin keine wesentliche Rolle. Die Makrotheorien von Talcott Parsons in Harvard stehen der im Entstehen begriffenen empirischen Sozialforschung von Paul Lazarsfeld an der Columbia University in New York gegenüber. In diesem Milieu schreibt der amerikanische Soziologe Robert King Merton (*1910 - †2003) seine Dissertation in Harvard über die Entwicklung der Wissenschaften im England des 17. Jahrhunderts (Merton 1938) und beginnt damit seine Karriere als erster ausgewiesener Wissenschaftssoziologe.

Dabei ist Mertons Forschung keineswegs auf die Untersuchung von Wissenschaft beschränkt. Schon in seiner Dissertation verfolgte er eine ähnliche Problematik, wie sie auch Fleck aufgeworfen hatte (WISSENSCHAFTSTHEORIE): es ging ihm um die Analyse eines Denkstils, der für die Epoche der frühen Moderne generell charakteristisch war. Auch seine Ar- 
beiten zu Struktur, Anomie und Devianz sind bis heute maßgebend und seine Konzepte der »sich selbst erfüllenden Prophezeiung« und des »Matthäus Effekts ${ }^{3}$ Allgemeinwissen. Seine Beiträge zur Wissenschaftsforschung hingegen werden heute respektiert, aber auch sehr kritisch beurteilt. Man könnte sogar sagen, dass die Wissenschaftsforschung in den späten 1970er Jahren bewusst von Merton Abstand genommen hat. Das geschah zum einen, weil man seine Analysen von Wissenschaft keineswegs uneingeschränkt teilte. Zum anderen musste man sich bewusst von der ungemeinen Dominanz der Merton'schen Ansätze befreien, um Raum für neue Entwicklungen zu schaffen. Dabei darf man allerdings nicht aus den Augen verlieren, dass Merton selbst diesen neuen Entwicklungen mit seinen Untersuchungen und Perspektiven entscheidend den Weg bereitet hat. Es ist daher sicherlich mehr als angemessen, seinen Untersuchungen und Konzepten trotz aller berechtigten Kritik achtsam gegenüberzutreten.

Stephen Cole, ein langjähriger Student und Kollege von Robert Merton, schreibt 2004 in einem Nachruf auf Merton im Sonderheft des Journals Social Studies of Science:

»Merton appeared to be an extreme workaholic and would, notoriously, rise at 4:30 and be at work at 5:00 so he could do the most important part of his work early in the morning when he was at his brightest. He would finish his writing and then go on to his teaching, thus saving his consulting for the late afternoon when he was tired and needed wits less then when writing. And to our knowledge as students, since we never got to see him at home, this pattern was followed seven days a week.«

3 »Sich selbst erfüllende Prophezeiung« bezeichnet das Phänomen, dass sich Vorhersagen häufig erfüllen, weil die Vorhersagenden sich dementsprechend verhalten, ohne sich dessen bewusst zu sein. Der Name des Matthäus Effekts geht auf das Matthäusevangelium zurück: »Denn wer da hat, dem wird gegeben werden, dass er Fülle habe; wer aber nicht hat, von dem wird auch genommen, was er hat.« - Mt 25,29 LUT Er bezeichnet das Phänomen der positiven Rückkopplung ursprünglich untersucht an Zitationshäufigkeiten von wissenschaftlichen Aufsätzen. Bekannte Autoren werden öfter zitiert als unbekannte. Es findet also eine zentrierende Bewegung statt. Gleiches gilt auch für andere Felder, z. B. für die Verteilung von Kapital und Vermögen in westlichen Gesellschaften. Zu den Konzepten Anomie und Devianz, siehe vor allem Merton (1957). 
Es ist wohl nicht unfair zu sagen, dass Merton als Mensch wie als Wissenschaftler eine nicht allseits und immer beliebte Persönlichkeit war. Glaubt man seinen Schülerinnen und Schülern, Kolleginnen und Kollegen, dann stellt sich Merton als ein kreativer Wissenschaftler dar, der bis zum Starrsinn an seinen Ideen festhielt. Dieses Beharren auf einmal formulierten Konzepten dürfte maßgeblich dazu beigetragen haben, dass seine Ansichten nicht den Wandel der Zeit mitgemacht haben, sondern bald in ein gewisses Abseits gerieten. Gerade diese konzeptionelle Schärfe und Pointierung jedoch ist es auch, die seine Konzepte bis heute als klar formulierte Reibungsfläche für alternative Ideen anbietet.

\section{Mertons deskriptive Seite}

Mertons Dissertation befasste sich mit dem Zusammenhang zwischen Puritanismus und Wissenschaft, im England des 17. Jahrhunderts. In ihrem ersten Teil machte Merton auf der Basis einer quantitativen Analyse der $\mathrm{Na}$ tional Biography Database sichtbar, dass Wissenschaft im dritten Viertel des 17. Jahrhunderts einen deutlichen Entwicklungssprung vollzog. Er begründete dies, in bewusster Anlehnung an die Religionssoziologie Max Webers (Weber 1934), mit dem zunehmenden Einfluss einer puritanischen Gesinnung. Was Weber für den Zusammenhang zwischen Puritanismus und Wirtschaft herausgearbeitet hatte, zeigte Merton für den Zusammenhang zwischen Puritanismus und Wissenschaft. Er argumentierte, dass die Puritaner ihre wachsende Macht auf mindestens drei Wegen absicherten: (1) durch ihre positive Gesinnung gegenüber Wissenschaft und Technologie, die ihre Macht widerspiegelten und gleichzeitig zu mehren versprachen; (2) durch ihren glühenden Glauben an Fortschritt, der in direktem Zusammenhang mit ihrer wachsenden sozialen wie ökonomischen Rolle stand; (3) durch ihre Feindseligkeit gegenüber den damaligen feudalen Herrschaftsstrukturen, die ihrer Beteiligung an politischer Kontrolle und Macht im Wege standen.

Der zweite Teil der Mertonschen Dissertation befasste sich mit einer Analyse der Frage, wie Wissenschaftler Themen auswählen und Aufmerksamkeit fokussieren. Es gelang ihm zu zeigen, dass es in der Hauptsache die praktischen Angelegenheiten der jeweiligen Zeit sind - wie z. B. das Problem der Schiffsnavigation im England des 17. Jahrhunderts - die den Auswahlprozess relevanter Probleme steuern. Dass der Prozess der Themen- und Problemwahl als ein sozialer und historisch eingebetteter Prozess 
zu untersuchen ist, der maßgeblich durch Faktoren außerhalb von Wissenschaft angetrieben wird, wird durch Merton zu einem Standardtopos der Wissenschaftssoziologie. (Cole 2004: 836 ff.) Vor allem der Schritt, den Merton vom ersten zum zweiten Teil seiner Dissertation vollzog, ist aus anthropologischer Sicht bemerkenswert. Während er im ersten Teil das Entstehen einer spezifischen gesellschaftlichen Organisationsform - die Wissenschaft - noch mit »dem Puritanismus« begründete, d. h. dem anthropologisch fragwürdigen Postulat einer homogenen Wertegemeinschaft, arbeitete er im zweiten Teil mit einer Begründung, die deutlich näher an einem anthropologischen Praxisbegriff liegt. Während er im ersten Teil noch die puritanische Gesinnung verantwortlich macht für eine spezifische Entwicklung der Wissenschaft, lenkt er den Blick im zweiten Teil auf die "praktischen Angelegenheiten«, die seines Erachtens Blick-, Denk- und Handlungsperspektiven der Wissenschaftler steuern. Mit der Erforschung des Einflusses dieser praktischen Kontexte auf Wissenschaft und Erkenntnis bereitete Merton den Weg für die Entwicklung der STS. Bis heute ist diese Frage ein zentraler Bestandteil der empirischen Wissenschaftsforschung.

Merton ebnet also den Weg für die Untersuchung von Wissenschaft als gesellschaftliche Institution. Dabei kommen zunächst die Strukturen von Wissenschaft selbst in den Blick. So schreibt er bereits 1938, dass die Wissenschaft als Institution - einmal etabliert, wie z. B. in Großbritannien die Royal Society Mitte des 17. Jahrhunderts - zweierlei Anziehungskräfte auf ihre Mitglieder ausübe, nämlich »generally prized opportunities of engaging in socially approved patterns of association with one's fellows and the consequent creation of cultural products which are esteemed by the group." (Merton, 1938: 231) Entscheidend ist hier, dass wissenschaftliche Erkenntnis als Ergebnis der Erfüllung sozialer Bedürfnisse - Anerkennung und rollenkonformes Verhalten - ihrer Protagonisten und nicht als Resultat eines quasi naturwüchsigen Strebens nach Wahrheit und Erkenntnis erscheint. An diese frühen Arbeiten Mertons knüpfen bis heute zahlreiche Studien der Wissenschaftssoziologie und -politik an, die versuchen, wissenschaftliches Arbeiten durch strukturelle und funktionale Indikatoren zu erklären: soziale Netzwerke, Zitationskartelle, Belohnungsstrukturen, Förderstrukturen, Hierarchien, Kooperationsvereinbarungen, usw. So ziemlich jeder Aspekt der Institution Wissenschaft ist bis heute auf die eine oder andere Art und Weise operationalisiert und untersucht worden. Vor allem große Trends und 
Entwicklungen auf der Makroebene lassen sich auf diese Art und Weise sehr gut erfassen und analysieren. ${ }^{4}$

Merton hat mit seinen Arbeiten aber nicht nur den Untersuchungen von institutionellen Strukturen in Wissenschaft den Weg gebahnt. Bemerkenswerter aus kulturanthropologischer Sicht ist die Tatsache, dass Merton bereits Ende der 1930er Jahre Wissen nicht als ein abstraktes Gut definierte, sondern als ein Kulturprodukt. Zwar verstand er dies nicht so, wie man es heute nach der konstruktivistischen Wende zumeist auffasst. Merton blieb Positivist insofern, als er an realen Wissensfortschritt glaubte. Trotzdem betonte er schon früh gegenüber den etablierten wissenschaftstheoretischen Positionen die Relevanz des Entstehungskontextes von Wissen. Es ist daher eigentlich verwunderlich, dass es mehr als 30 Jahre dauerte, bis die Kultursoziologie und -anthropologie diese Perspektive aufgriff und weiterentwickelte. In den 1970er Jahren war es vor allem Pierre Bourdieu, der durch eine kritische Analyse der sozialen Bedingtheit von Wissenschaft diese als spezifisches Feld darstellte und damit ebenfalls unterstrich, auf welche Art und Weise wissenssoziologische Untersuchungen fruchtbare Ansätze für allgemeinere Sozialtheorien bereithalten können. (Bourdieu 1975)

Merton untersuchte also nicht nur die Struktur von Wissenschaft, als Institution, sondern auch ihr Verhältnis zur Gesellschaft. Vorsicht ist bei der Lektüre vieler seiner Schriften insofern geboten, als dass Merton, wie zuvor bereits Kuhn, nur unzureichend Deskription und Präskription voneinander trennt $^{5}$, d. h. Mertons Schriften enthalten häufig Anteile deskriptiver soziologischer Analyse, vermengen diese aber mit normativen Aussagen über die Struktur von Wissenschaft, wie sie nach Meinung Mertons sein sollte. Aus dieser Verquickung entstehen vielfältige Probleme, auf die hier im Einzelnen nicht eingegangen werden muss. Stephen Cole springt Merton posthum zur Seite, wenn er schreibt: »Merton chose not to take the route of examining how social factors influenced specific pieces of science; rather, he was

4 Diese Formen der Wissenschafts- und Hochschulforschung sind nicht Bestandteil des vorliegenden Bandes, da dieser sich vor allem praxistheoretisch orientiert und auf ethnographische Mikro-Studien fokussiert. Einen guten Einstieg in die makrologische Wissenschafts- und Hochschulforschung vermitteln z. B. Hornbostel (2006) und Simon (2001).

$5 \mathrm{Ob}$ dies (wissenschafts-)politische Absicht ist oder lediglich die Folge einer unzureichenden empirischen Dichte, sei hier dahingestellt. Eine detaillierte Diskussion dieser Ambivalenz in Mertons Analysen findet sich bei Paul Feyerabend (1990). 
interested in how social factors influenced science in a broader sense (for example, the influences on the foci of attention of scientists in England during the last part of the 17th century)." (Cole 2004: 842 ff.) Eine kritischere Lesart, und dies zeigen die folgenden Beiträge dieses Bandes, macht deutlich, dass detaillierte historische, wie soziologische und ethnographische Untersuchungen des Einflusses sozialer Faktoren auf Wissenschaft Mertons Befunde zumindest in ihrer Generalität in Frage stellen.

\section{Mertons präskriptive Seite}

Konzentriert man sich auf die normativen Elemente von Mertons Analysen, so kommt man nicht umhin, sein sehr positivistisches Verständnis von Wissenschaft und Erkenntnisgewinn zu bemerken. Wie Cole zurecht hervorhebt (Cole 2004: 842 ff.), machte Merton in der annotierten Version seines Aufsatzes von 1942 zur gesellschaftlichen Bedingtheit von Wissenschaft deutlich, dass er der Meinung war, die Soziologie könne die Inhalte der Wissenschaft nicht erklären, da diese letztlich durch die Natur selbst determiniert würden. In einem Kommentar der damaligen Entwicklungen in der Sowjetunion, die seines Erachtens Wissenschaft als »national in form and class in content « darstellten, schreibt Merton:

»This view confuses two distinct issues: first the cultural context in any given nation or society may predispose scientists to focus on certain problems, to be sensitive to some and not other problems on the frontiers of science. This has long since been observed. But this is basically different from the second issue; the criteria of validity of claims to scientific knowledge are not matters of national taste and culture. Sooner or later, competing claims to validity are settled by the universalistic facts of nature, which are consonant with one and not with another theory." (1957 [1949]: 554)

In einem Nachdruck des Essays 1973 ändert Merton zwar den letzten Satz »sooner or later, competing claims to validity are settled by universalistic criteria« (Merton 1942/1973: 271) - aber diese Verschiebung von »universalistic facts of nature« hin $\mathrm{zu} »$ universalistic criteria« ändert seine Grundposition nur unwesentlich. Diese positivistische Position, die immer noch starke Anklänge an den ersten Wiener Kreis und Karl Popper offenbart, muss man bei der Lektüre von Mertons Überlegungen zum Zusammenhang von Wissenschaft und Gesellschaft im Hinterkopf behalten. Merton defin- 
iert vor diesem Hintergrund vier institutionelle Gebote, die die Position von Wissenschaft in der Gesellschaft sowie ihren Ethos bestimmen sollen:

\section{Universalität}

»Aussagen mit Wahrheitsanspruch müssen vorher festgesetzten unpersönlichen Kriterien ${ }^{6}$ genügen, die in Einklang mit Beobachtung und mit bereits bestätigtem Wissen stehen. « ${ }^{7}$ (Merton 1942/1973: 271f.) Objektivität schließt Partikularismen aus; hieraus folgte für ihn zudem, dass Karrierepfade Talentierten ohne Unterschied offen stehen müssten.

\section{Kommunismus}

Kommunismus versteht sich bei Merton nicht eigentlich politisch, sondern im erweiterten Sinne als die gemeinsame Eigentümerschaft an Waren. Merton konstatierte, dass der Anspruch des Wissenschaftlers auf >sein` geistiges >Eigentum darauf beschränkt bleiben solle, Anerkennung und Achtung zu erzeugen. »Aus der institutionellen Konzeption von Wissenschaft als Teil der öffentlichen Domäne folgt auch das Gebot, Ergebnisse zu veröffentlichen. Geheimhaltung ist die Antithese dieser Norm.« (ebd.: 274) Und weiter: »Die Bescheidenheit des wissenschaftlichen Genies ist nicht nur kulturell angemessen, sondern resultiert aus der Erkenntnis, dass wissenschaftlicher Fortschritt die Zusammenarbeit von vergangenen und gegenwärtigen Generationen erfordert.« (ebd.: 275). Merton betonte mit feiner

6 In diesem Kontext steht auch Mertons Auseinandersetzung mit der MarxistischLeninistischen Position zu Wissenschaft: »Only a cosmopolitan without a homeland, profoundly insensible to the actual fortunes of science, could deny with contemptuous indifference the existence of the many-headed national forms in which science lives and develops. In place of the actual history of science and the concrete paths of its development, the cosmopolitan substitutes fabricated concepts of a kind of supernational, classless science, deprived, as it were, of all the wealth of national coloration, deprived of the living brilliance and specific character of a people's creative work, and transformed into a sort of disembodied spirit. [...] Marxism-Leninism shatters into bits the cosmopolitan fictions concerning supraclass, non-national, suniversal science, and definitely proves that science, like all culture in modern society, is national in form and class in content." (Editorial in Voprosy filosofii no.2 1948; transl. in Current Digest of the Soviet Press 1 (1), 1949, zitiert in Merton 1973: 271)

7 Übersetzung dieses und der folgenden Zitate durch JN. 
Ironie, dass Wissenschaftler häufig einen professionellen Habitus an den Tag legen würden, der die Originalitätsansprüche ihrer Arbeit herausstelle und dabei die Einbettung der >eigenen`Gedanken in >fremdeく übersehe. (Merton 1965)

\section{Uneigennützigkeit [disinterestedness]}

Mit Uneigennützigkeit bezeichnete Merton kein Persönlichkeitsmerkmal, auch wenn diese Norm oft ausgelegt wird als ein Plädoyer für altruistisches Verhalten und gegen Missbrauch in der wissenschaftlichen Praxis und die Privatisierung von Wissen. Vielmehr ging es im Kern darum, dass Wissenschaft die Nachprüfbarkeit von Ergebnissen beinhalte und wissenschaftliche Arbeit damit immer einer genauen Überprüfung durch Kollegen gerecht werden müsse. »Die Übersetzung der Uneigennützigkeitsnorm in die Praxis, wird letztlich genau dadurch unterstützt, dass Wissenschaftler ihren Kollegen gegenüber rechenschaftspflichtig sind. Hier stehen Gemeinschaftsgefühl und Zweckmäßigkeit miteinander im Einklang.« (Merton 1965: 276)

"It is probable that the reputability of science and its lofty ethical status in the estimate of the layman is in no small measure due to technological achievements. Every new technology bears witness to the integrity of the scientist. Science realizes its claims.« (ebd.: 277)

\section{Organisierter Skeptizismus}

Organisierter Skeptizismus stellte einen klaren Bezug zu Poppers Falsifikationismus her und forderte das ständige Hinterfragen neuer Erkenntnisse, aber auch etablierter Gewissheiten. Merton betonte, dass organisierter Skeptizismus sowohl einen methodologischen wie institutionellen Auftrag darstelle, d. h. für jedes einzelne Mitglied der Wissenschaftsgemeinde im Alltag gelten solle, wie auch für das Feld als Ganzes.

Diesen vier institutionellen Geboten nun sollte Wissenschaft folgen: Universalität, Kommunismus, Uneigennützigkeit, organisierter Skeptizismus. Aus heutiger Sicht scheint offensichtlich, dass dies keine Beschreibung und Analyse von Wissenschaft sein kann, wie sie tatsächlich praktiziert wird. Und auch 1940 und im Umfeld ehrenwerter Institutionen wie der Royal So- 
ciety und der Ivy League Universitäten wird deutlich gewesen sein, dass Anspruch und Wirklichkeit hier nicht immer Deckungsgleichheit erreichten. Merton selber kokettierte in seinem essayistischen Werk »On the shoulders of giants« mit den etablierten Vorstellungen von Erkenntnisgewinn, Lernen und akademischem Habitus und zeigte damit, dass er sich mehr als bewusst war, dass Erkenntnisgewinn immer verschlungeneren Pfaden folgt und folgen muss, als mit vier normativen Imperativen zu erklären und zu bestimmen wäre. (Merton 1965) Seine eigene Karriere und Arbeitsweise verkörperte diese Diskrepanz bis zu einem gewissen Grad. Denn einerseits stellte er in seinen Werken immer wieder heraus, dass Erkenntnisgewinn eine kollektive Errungenschaft sei, die weder als abstrakter Prozess, noch als Akt individuellen Genius' verstanden werden dürfe. Dabei betonte er vor allem den inter-generationalen Aspekt und räumte einer synchronen Perspektive auf Kollektivität weniger Raum ein als dies beispielsweise die Analysen eines Ludwik Fleck von Denkstilen und - kollektiven getan hatten. Andererseits trug seine eigene Arbeitsweise doch stark individualistische Züge. Das ist formal keine Paradoxie. Auch meinungsstarke Personen können sich selbstverständlich mit Vorgängern und Kollegen auseinandersetzen. Jedoch kann man nicht umhin, zwischen Mertons eigener Arbeitsweise und seinem normativen Verständnis von Wissenschaft immer wieder einen gewissen Widerspruch zu entdecken.

Wichtiger als Mertons persönliche Arbeitsweise ist jedoch, dass Wissenschaft als Institution in der ersten Hälfte des 20. Jahrhunderts noch nicht die zentrale Stellung im Spannungsfeld von Politik, Wirtschaft und Gesellschaft innehatte, die sie bald darauf, maßgeblich nach dem 2. Weltkrieg und im Zuge des Kalten Krieges, erreichte. Dies wird zum Beispiel deutlich am Bild des charismatischen Führungsstils, das Max Weber noch zu Beginn des Jahrhunderts entwerfen konnte. (Weber 1922/2002) In diesem Bild konsultieren Politiker zwar Wissenschaft - schließlich handelt es sich um moderne, fortschrittliche Gesellschaften - sie sind aber in ihren Entscheidungsmöglichkeiten nicht in vergleichbarer Weise auf wissenschaftlichen Rat angewiesen, wie das heute der Fall ist. Umgekehrt gilt, dass Wissenschaft als Institution, in der Zeit, in der Merton seine Hauptwerke verfasste, noch nicht in dem Maße mit Versuchen der Inanspruchnahme durch außerwissenschaftliche Interessen konfrontiert war, wie dies für die Nachkriegszeit festgestellt werden muss. 


\section{WISSENSCHAFTS- UND TECHNOLOGIEFORSCHUNG IN DEN NACHKRIEGSJAHREN}

In den 1950 und 60er Jahren ändert sich dies in signifikanter Weise. Wissenschaft rückt zunehmend ins Zentrum politischer Strategieentwürfe. Dies wird zum einen angetrieben durch eine zunehmend wissens- und technologieintensive industrielle Entwicklung, die zu gezielten privaten Investitionen in Wissenschaft führt. Zum anderen, und zumindest zu Beginn dieser Entwicklung wichtiger, nimmt der Grad der Militarisierung politischer Strategien stetig zu. Das schlägt sich auf beiden Seiten des Eisernen Vorhangs in gigantischen Investitionen in militärische Forschung und Entwicklungen nieder. Große Förderprogramme des US-Amerikanischen Verteidigungsministeriums konzentrieren sich auf Technologieentwicklung. Dabei darf nicht übersehen werden, dass auch die scheinbar >harmlosen $<$ Wissenschaften, d. h. die >weichen $<$ Sozial- und Kulturwissenschaften, von dieser Entwicklung massiv betroffen und beeinflusst waren.

So spielte gerade die Kulturanthropologie in diesem Kontext eine wichtige Rolle, da sie doch rascher als mancher vermutet hätte dazu bereit war, ihre ausgeprägten regionalen, kulturellen und historischen Kompetenzen in den Dienst strategischer Interessen amerikanischer Außenpolitik zu stellen. (Vgl. Rabinow et al. 2008) Dabei handelte es sich seltener um Auftragsforschung in dem trivialen Sinne. Vielmehr waren Großprogramme, wie z. B. die human relations area files (HRAF) seit 1949 an der Yale University angesiedelt $^{8}$, natürlich immer auch, und vielleicht auch zuerst, Programme, die auf wissenschaftliche Erkenntnis orientiert waren; im Falle der HRAF zur vergleichenden und quantifizierenden Analyse, Kartierung und Katalogisierung > fremder Kulturen. Jedoch kamen die Gelder für diese Forschung immer wieder aus dem Verteidigungsministerium. Bevor dieses Kapitel die Reaktion der amerikanischen Wissenschaftssoziologie und -philosophie auf diese veränderte Konstellation ein wenig genauer in den Blick nimmt, um damit den Übergang von einer Wissenschafts- zu einer Wissenssoziologie und damit die Geburt der Science and Technology Studies im heutigen Sinne zu beleuchten, sei an dieser Stelle in aller Kürze die Konfiguration in der Bundesrepublik Deutschland der 1960er Jahre skizziert.

8 Siehe z. B. Naroll et al. (1976). 
Das Ende des Wirtschaftswunders am Übergang zu den 1960er Jahren und der Bau der Mauer 1961 charakterisierten das politische und wirtschaftliche Milieu in der Bundesrepublik Deutschland, in dem sich ein Zeitgeist manifestierte, der wesentlich durch »wissenschaftlich-technische Naturbeherrschung und wissenschaftliche Politikberatung zur rationalen Steuerung von Individuen, Gesellschaft und Ökonomie« ausgerichtet war. (Vgl. Stapelfeld 2005) Die als Positivismusstreit in die Geschichte eingegangene Auseinandersetzung zwischen Karl Popper und Theodor Adorno bzw. in der Folge zwischen Hans Albert und Jürgen Habermas, hatte die Idee der Möglichkeit des Erkenntnisgewinns mittels Induktion endgültig verabschiedet. Beobachtung sei, da waren sich die Kontrahenten einig und folgten Poppers im kritischen Rationalismus formulierten Ansichten, niemals vortheoretisch, sondern immer bereits theoriegeleitet. Popper gestand seinen Streitpartnern zu, dass theoriegeleitet zwar für individuelle Wissenschaftler auch immer interessengeleitet bedeute, er vertrat jedoch die Auffassung, dass Interessenorientierung sich über einen längeren Zeitraum verlöre, da Wissenschaft als Institution dafür sorge, dass Theorie und Welt sich einander annäherten. Die kollektive Praxis der Wissenschaft würde, so Popper in einer quasi-evolutionären Argumentation, über Zeit die interessengeleiteten Aspekte von Theorie ausselektieren und damit eine Annäherung von Theorie an Wahrheit befördern.

Adorno verstand diese Sicht auf Wissenschaft und Gesellschaft letztlich als der Utopie technischer Weltbeherrschung verfallen. (vgl. Tiedemann/ Adorno 1990) Tatsachen waren für ihn die Verdinglichungen des Gesellschaftlich-Allgemeinen. Theorie könne sich daher nicht den Tatsachen annähern, d. h. nach Wahrheit im kritisch-rationalistischen Sinne streben, da sie immer schon in ihnen enthalten sei. Anders als bei Popper müssten Tatsachen selbst daher notwendig als Produkt gesellschaftlicher Bedingungen gedeutet werden. Theorie im Popperschen Sinne dupliziere bloß die Tatsachenwelt. Das könne nicht das Ziel sein. Vielmehr, so Adorno, müsse diese Tatsachenwelt überschritten und dadurch erkannt werden, dass Kritik »nicht auf wahre Theorie, sondern auf eine >wahre`, eine bewusste Gesellschaft ihrer selbst bewusster Individuen« ziele. (Stapelfeld 2005: 143) Jürgen Habermas wendete Adornos Argumentation noch einmal philosophisch-anthropologisch und schaffte damit die Möglichkeit, innerhalb von Kommunikation die Utopie der herrschaftsfreien Gesellschaft zu denken. (Vgl. u. a. Habermas 1968) 
Für den Kontext dieses Kapitels sind zwei Aspekte dieser grundlegenden Debatte der deutschen Nachkriegszeit wichtig. Sie verdeutlicht erstens, wie die Erfahrungen des Dritten Reiches in Deutschland das Verständnis des Zusammenhangs von Wissenschaft, Gesellschaft und Erkenntnismöglichkeiten geprägt haben. Wissenschaft und Gesellschaft werden als viel fundamentaler verschränkt begriffen, als dies in der anglo-amerikanischen Wissenschaftssoziologie der Fall ist. Allerdings verläuft die Debatte im deutschsprachigen Raum deutlich philosophisch-abstrakter und verliert rasch die konkreten Bedingungen von Wissensproduktion aus dem Blick. Zweitens ist der Positivismusstreit eng verzahnt mit einer zweiten für die Wissenschafts- und Technikforschung in Deutschland prägenden Auseinandersetzung: der Technokratiedebatte. Diese wurde ebenfalls am Übergang zu den 1960er Jahren ausgelöst, diesmal vor allem durch kulturkritische Beiträge von Arnold Gehlen und Helmut Schelsky. (Gehlen 1957, Schelsky 1965)

Im Kern dieser Debatte stand die Vorstellung, dass Technologie und technologische Entwicklung den Bereich des sozialen und natürlichen Kosmos unter eine technokratische, d. h. rational-totalitäre Herrschaft subsumieren würde. In einer zunehmend ökonomisch dominierten Gesellschaft, in der Automatisierung und Perfektionierung zentrale Triebkräfte darstellten, war es den Verfechtern der Technokratiekritik nicht vorstellbar, wo moralische Entscheidungen überhaupt noch ansetzen sollten. Man sah Gesellschaft als politisches, moralisches und soziales Gebilde grundlegend in Gefahr. Diese Debatte offenbart eine wichtige Prägung durch die frühe deutsche philosophische Anthropologie, denn sie positioniert unmenschlich-rationale Technik gegen ein zutiefst menschliches, von sunnatürlichen Einflüssen freies und immer ein wenig romantisches Menschenbild. Hier setzt, wie das Kapitel zur SOZIALEN KOnSTRUKTION vON TECHNOLOGIE in diesem Band zeigt, in den 1970er Jahren die Techniksoziologie in Deutschland an, die sich Technik deutlich empirischer zuwendet und damit zunehmend ein zutiefst menschliches Verständnis von Technik zu prägen beginnt. (z. B. Weingart 2003, Joerges 1987, Maasen 2009, Rammert 1988)

Positivismusdebatte und Technokratiedebatte prägen also die Analyse von Wissen, Wissenschaft und Technologieentwicklung in der Bundesrepublik Deutschland in den 1950er, 60er und frühen 70er Jahren. Analysen von Wissenschaft und Technologieentwicklung werden hier immer notwendig und explizit auch als Gesellschaftstheorie und -kritik verstanden. 
Ihre Grundlage sind Verständnisse von gesellschaftlicher Entwicklung und ihren moralischen Dimensionen, die selten auf detaillierten empirischen Studien fußen. Sie sind damit aber ein wichtiger theoretischer Wegbereiter für eine Verschiebung in der Wissenschaftssoziologie, die nun im Folgenden anhand der anglo-amerikanischen Entwicklung dieses Feldes in den 1960er und 1970er Jahren skizziert wird und die als direkter Vorläufer der Science and Technology Studies verstanden werden kann.

\section{0er Jahre: Wissenschaft unter Druck}

Im anglo-amerikanischen Raum änderte der Beginn des Kalten Krieges die Rolle von Wissenschaft in Gesellschaft. Jerome Ravetz - Wissenschaftsphilosoph, Vater des Begriffs post-normal science und selbst betroffen von der anti-kommunistischen Linie der McCarthy Ära beschrieb Ende der 1960er Jahre sehr gut die neue Stimmung, in der zunehmend die Sorge über eine staatliche und wirtschaftliche Vereinnahmung von Wissenschaft zum Ausdruck kam:

»The activity of modern natural science has transformed our knowledge and control of the world about us; but in the process it has also transformed itself; and it has created problems, which natural science alone cannot solve. Modern society depends increasingly on industrial production based on the application of scientific results; but the production of these results has itself become a large and expensive industry. All this has happened so quickly within the past generation, that the new situation, and its implications are only imperfectly understood. [...] The illusion that there is a natural science standing pure and separate from all involvement with the society is disappearing rapidly; but it tends to be replaced by the vulgar reduction of science to a branch of commercial or military industry. Unless science itself is to be debased and corrupted, and its results used in the headlong rush to social and ecological catastrophe, there must be a renewed understanding of the very special sort of work, so delicate and so powerful, of scientific inquiry.

Science is no longer a marginal pursuit of little practical use carried on by a handful of enthusiasts; and it no longer needs to justify itself by a direct answer to the challenge of other fields of knowledge claiming exclusive access to truth. As the world of science has grown in size and in power, its deepest problems have changed from the epistemological to the social. ... Before an understanding of this extended and enriched science, we must consider those sorts of disciplined inquiry whose goals 
include power as well as knowledge. [...] Science is so important, and expensive, that the major policy decisions concerning its development are increasingly being taken by the state, rather than being left to the judgement of the scientists and their private patrons. Accordingly, the progress of science becomes a matter of politics.«

Hier wird sehr deutlich, dass die Bedenken der Wissenschaftsphilosophen der ersten Hälfte des 20. Jahrhunderts, nämlich die Abgrenzung der Wissenschaft (sprich der Naturwissenschaft) von der Philosophie, nun in den Hintergrund tritt. Stattdessen muss sich Wissenschaft zunehmend in Abgrenzung zur politischen Sphäre definieren. ${ }^{9}$ Die Fragen an sie sind nicht nur noch epistemologischer, sondern zunehmend auch sozialer und explizit politischer Natur. Dabei rückte Ravetz zwei Aspekte in den Vordergrund, die bis heute in der Wissenschaftsforschung eine zentrale Rolle spielen:

Erstens die Tatsache, dass Wissenschaft keineswegs als eine vorsoziale Suche nach Erkenntnis verstanden werden kann, sondern als ein Prozess untersucht werden muss, der auf mannigfaltige Art und Weise mit gesellschaftlicher Praxis verwoben ist. Hier zeigen sich deutliche Parallelen zu den oben skizzierten Debatten in Deutschland und hier liegen auch wichtige Wurzeln für die feministische Kritik, die in den 1980er Jahren und 1990er Jahren vor allem die kulturanthropologische Wissenschaftsforschung entscheidend geprägt hat. So greift die US Amerikanische Kulturanthropologin Emily Martin (1997; 1998) beispielsweise dieses ineinander VerwobenSein von Wissenschaft und Gesellschaft in einem in der Wissenschaftsforschung viel beachteten Aufsatz auf, um aus anthropologischer Sicht deutlich zu machen, dass Wissenschaft nicht als Elfenbeinturm zu verstehen sei, der in und über Gesellschaft throne, sondern dass Wissenschaft wie ein Fadenspiel untrennbar mit Gesellschaft verwoben sei und als integraler Bestandteil von kultureller Praxis untersucht werden müsse. (Martin 1997)

Zweitens wies Ravetz zu Recht daraufhin, dass Wissenschaft in den 1960er Jahren längst kein exotisches Feld einiger weniger Enthusiasten mehr darstellte, sondern eine in Größe und Wirkmacht ständig wachsende Domäne mit enormer wirtschaftlicher, politischer und gesellschaftlicher Bedeutung. Wissenschaftliches Wissen wurde hier als zentrale gesellschaft-

9 Zur Problematik des Politikbegriffs in der Wissenschaftsforschung siehe STS UND POLITIK. 
liche Ordnungskraft konzipiert, dessen Analyse im Zentrum von Sozialund Kulturforschung angesiedelt sein sollte. Die damit in engem Zusammenhang stehende unmittelbare und unwiderrufliche Verknüpfung von Wissen und Macht bereitete den Weg für Michel Foucaults Konzept des Macht-Wissens und etablierte damit endgültig Wissen und Wissenschaft als politisch im dem sub- oder mikropolitischen Sinne eines Ulrich Beck oder Bruno Latour. (Foucault 1972, 1976; Beck 1993; Latour 2005)

Im Laufe der 1960er Jahre begannen eine Reihe kritischer Beobachter der gesellschaftlichen Entwicklungen, wie der ungarische Chemiker und Philosoph Michael Polanyi und der österreichische Wissenschaftsphilosoph und -theoretiker Paul Feyerabend (1976), vor einer Vereinnahmung der Wissenschaft durch staatliche oder wirtschaftliche Interessen zu warnen. Aus diesen Arbeiten erwuchs unter anderem eine Neuformulierung der frühen Mertonschen Gebote an den wissenschaftlichen Ethos, um die Autonomie der Wissenschaft wieder zu stärken. Polanyi veröffentlichte bereits 1962 seinen berühmt gewordenen Aufsatz The Republic of Science, in dem er Wissenschaft als engmaschiges Netzwerk von Wissenschaftlern beschrieb, deren Kompetenzen überlappten. Diese Überlappungen ermöglichten gegenseitige Kontrolle und Aufsicht. Niemand arbeite völlig isoliert, sondern immer zu einem gewissen Grad in Kompetenzfeldern anderer Forschender und damit auch unter ihrer Kontrolle. Wissenschaftlicher Verdienst, schrieb Polanyi, würde nach Plausibilität, wissenschaftlichem Wert (Genauigkeit, Bedeutung für das System, intrinsische Attraktivität des Themas) und Originalität beurteilt. Die Autorität, dies zu beurteilen liege dabei, und das ist entscheidend, zwischen den Forschenden und nicht $\ddot{u} b e r$ ihnen. (Polanyi 1962: 4ff.) Die Kriterien für wissenschaftliche Qualität und Güte ergäben sich aus der wissenschaftlichen Praxis und Organisation selbst, residierten also im Netzwerk Wissenschaft. Sie könnten und sollten nicht außerhalb von wissenschaftlicher Praxis entwickelt und begründet werden, um dann Wissenschaft zu steuern.

Mit dieser Analyse übernahm Polanyi im wesentlichen Mertons Gebote, lokalisierte aber die Autorität zur Beurteilung, ob diesen Geboten ausreichend Folge geleistet werde, explizit in Wissenschaft selbst. Er erteilte damit jeglicher Form von äußerer Steuerung und Ausrichtung von Wissenschaft eine klare Absage. Politische und wirtschaftliche Maßgaben seien den wissenschaftsinternen Kriterien und Maßstäben immer nachzuordnen. 
Ebenfalls wies er in diesem Zusammenhang darauf hin, dass Wissenschaft auf Respekt im öffentlichen Diskurs angewiesen sei, um ungehindert voranschreiten zu können. Eine Wissenschaft, die öffentlichen Respekt verliere, werde ihren Freiraum einbüßen und damit notwendig externer Kontrolle unterworfen werden. Polanyi entwickelte diese Position allerdings nicht aus der Annahme heraus, dass Wissenschaft tatsächlich ein rein erkenntnisgeleitetes Unterfangen sei, sondern aus der Sorge heraus, dass Wissenschaft im Dienste einer anderen institutionellen Autorität nicht funktionieren könne:

»It appears that a society bent on discovery must advance by supporting independent initiatives, coordinating themselves mutually to each other. [...] Even so, all these independent initiatives must accept for their guidance a traditional authority, enforcing its own self-renewal by cultivating originality among its followers.« (Polanyi 1962: 20)

Und diese wissenschaftliche Arbeitsweise, so Polanyi, erzwingt ihre eigene Fortführung im Allgemeinen; gerade um ihre Subversion im Einzelfall zu kultivieren. (Ebd.: 17) Wir sehen hier durchaus noch Anklänge an das alte positivistische Bild von Wissenschaft des frühen 20. Jahrhunderts, erfahren aber bei Polanyi als einem der ersten auch bereits die Ausrichtung auf ganz neue Problemstellungen.

Die politischen Aspekte von Polanyis Linie griff Paul Feyerabend in seinem Buch »Wider den Methodenzwang« auf. (Feyerabend 1976) »Anything goes« ist seine berühmt gewordene Forderung für die wissenschaftliche Methode. Er argumentierte damit für einen Methodenpluralismus vor allem, weil ihm keine einzelne Methode absolut verbindliche Grundsätze für wissenschaftliches Arbeiten zu liefern schien und keine noch so gut begründete Regel nicht irgendwann bereits verletzt worden und damit Fortschritt erzielt worden wäre: »[Erkenntnis] ist ein stets anwachsendes Meer miteinander unverträglicher Alternativen; jede einzelne Theorie, jedes Märchen, jeder Mythos, der dazugehört, zwingt die anderen zu deutlicherer Entfaltung, und alle tragen durch ihre Konkurrenz zur Entwicklung unseres Bewußtseins bei.« (Feyerabend 1976) Diese Konkurrenz jedoch erfordere die intensive und systematische Auseinandersetzung mit vergangenen und gegenwärtigen Generationen, wie schon Merton und Polanyi gefordert hatten, und dürfe keineswegs mit Chaos oder Willkür gleichgesetzt werden. 


\section{FAZIT}

Statt Naturwissenschaft über die Unterscheidung von der Philosophie wissenschaftstheoretisch zu charakterisieren, ging es also in den 1960er und 70er Jahren vermehrt um eine kritische Untersuchung von Wissenschaft im Allgemeinen - nicht nur den Naturwissenschaften - als Institution in sozial und historisch spezifischen Kontexten. Damit gewann auch eine genauere Analyse wissenschaftlicher Praxis, ihrer Motive und Motivationen, ihres Arbeitsalltags und ihrer Rituale, und ihrer technologischen Manifestationen an Relevanz. Zugleich veränderte sich im Zuge des Kalten Krieges das Verhältnis von Wissenschaft, Politik, Wirtschaft und Gesellschaft grundlegend. Eine zunehmende Inanspruchnahme von Wissenschaft durch Politik und Wirtschaft stellte sich ein. Diese legitimierte zunächst weitere Untersuchungen der Produktionsbedingungen und -kontexte von Wissen und Technologie. Im Zentrum dieser Analysen stand nach wie vor Wissenschaft als Institution, wie es einer Mertonschen Wissenschaftssoziologie entspricht. Ziel war es, ungerechtfertigte Einflussnahmen von Gesellschaft auf Wissenschaft aufzuzeigen und sich so den nötigen Freiraum für wissenschaftliches Arbeiten gegen anderweitige Interessen zu erkämpfen. Diese Form der wissenschaftssoziologischen und wissenschaftspolitischen Arbeit hat bis heute eine wichtige Funktion und hat gerade in den letzten zehn Jahren wieder an Bedeutung gewonnen. Diskussionen um mode 2 science, Förderinstrumente des Bundes und der Europäischen Union, wie z. B. die Exzellenz-Initiative, oder neue Evaluationsverfahren von universitärer Forschung haben dazu beigetragen, dass Überlegungen zur Steuerung von Wissenschaft durch gesellschaftliche Interessen wieder neue Aktualität erlangt haben. (z. B. Nowotny et al. 2003)

In den 1970er Jahren kam aber eine weitere Frage auf, die dem Feld der Science and Technology Studies seine eigentliche Daseinsberechtigung verliehen hat: Welche Konsequenzen haben diese unterschiedlichen Steuerungsversuche und damit einhergehende Veränderungen von universitärer Infrastruktur eigentlich auf das Wissen selbst, das an diesen Orten produziert wird? Diese epistemologische Komponente war bis dahin völlig vernachlässigt worden, da Wissenschaft als Institution konzipiert, und damit ihr Innenleben weitgehend ausgeblendet worden war. Die positivistische Position des Wiener Kreises hatte bis in die 1970er Jahre nachgewirkt und verhindert, dass der epistemologische Gehalt und Status von wissenschaft- 
lichem Wissen zum Gegenstand sozialwissenschaftlicher Forschung wurde. Erst die zunehmende Politisierung von Wissenschaft und die Entzauberung wissenschaftlichen Wissens durch Positivismusstreit, Technokratiedebatte und die zunehmend kritischen Anmerkungen der amerikanischen Wissenschaftssoziologie machten deutlich, dass wissenschaftliches Wissen selbst sehr wohl Bestandteil soziologischer Analysen sein sollte.

Diese Erkenntnis markiert die Geburtsstunde der Science and Technology Studies, wie sie in diesem Band vorgestellt werden. Sozial- und kulturwissenschaftliches Interesse sollte sich nicht nur auf Wissenschaft richten, sondern auch auf Wissen: von der Wissenschaftssoziologie zur Soziologie wissenschaftlichen Wissens. In den späten 1970er und frühen 1980er Jahren, das zeigen die folgenden Kapitel, entwickelte sich aus diesem Interesse an wissenschaftlichem Wissen rasch eine breit gefächerte empirische Forschungslandschaft. Diese baute damals ganz entscheidend auf den theoretischen Debatten der 1960er Jahre auf, ging aber auf der Basis der empirischen Befunde bald deutlich über diese Debatten hinaus und problematisierte Wissenschaft als Praxis, als tatsächliche Arbeit in ganz neuer Weise. 


\section{LITERATUR}

Beck, Ulrich (1993): Die Erfindung des Politischen. Zu einer Theorie reflexiver Modernisierung, Frankfurt/M.: Suhrkamp.

Bourdieu, Pierre (1975): »The Specificity of the Scientific Field and the Social Conditions of the Progress of Reason«. Social Science. Information sur les sciences sociales XIV (6) 19-47.

Cole, Stephen (2004): »Merton's Contribution to the Sociology of Science«. Social Studies of Science 34(6), S. 829-44.

Dahms, Hans-Joachim (1994): Positivismusstreit: Die Auseinandersetzungen der Frankfurter Schule mit dem logischen Positivismus, dem amerikanischen Pragmatismus und dem kritischen Rationalismus, Frankfurt/M.: Suhrkamp.

Dewey, John (2008): Logik. Die Theorie der Forschung, Frankfurt/M.: Suhrkamp

Feyerabend, Paul (1970): „Consolations for the Specialist«. In: Alan Musgrave/Imre Lakatos (Hg.), Criticism and the Growth of Knowledge, Cambridge: Cambridge University Press, S. 197-230.

Feyerabend, Paul. Wider den Methodenzwang. Skizze einer anarchistischen Erkenntnistheorie, Frankfurt/M.: Suhrkamp.

Foucault, Michel (1972): »Truth and Power«. In: Colin Gordon (Hg.), Power/Knowledge: Selected Interviews and Other Writings 1972-1977, New York: Pantheon Books, S. 109-33.

Foucault, Michel (1976): Überwachen und Strafen. Die Geburt des Gefängnisses, Frankfurt/M.: Suhrkamp.

Gehlen, Arnold (1957): Die Seele im technischen Zeitalter. Sozialpsychologische Probleme in der industriellen Gesellschaft, Hamburg: Rowohlt.

Habermas, Jürgen (1968): Erkenntnis und Interesse. Frankfurt/M.: Suhrkamp.

Hornbostel, Stefan (2006): »Leistungsmessung in der Forschung. Von der Qualitätssicherung der Lehre zur Qualitätsentwicklung als Prinzip der Hochschulsteuerung«. Beiträge zur Hochschulpolitik 1, S 219-28.

Joerges, Bernward (1987): Technik, Umwelt, Alltag - Eine Bestandsaufnahme neuerer Soziologischer Forschung, Berlin: Wissenschaftszentrum. 
Knorr-Cetina, Karin (1981): The Manufacture of Knowledge - an Essay on the Constructivist and Contextual Nature of Science, Oxford: Pergamon Press.

Knorr Cetina, Karin (1999): Epistemic Cultures. How the Sciences make Knowledge, Cambrigde: Harvard University Press.

Kuper, Adam (1988): The Invention of Primitive Society: Transformations of an Illusion, London/New York: Routledge.

Kuper, Adam (2005): The Reinvention of Primitive Society: Transformations of a Myth, 2. Aufl., Milton Park/Abingdon/Oxon/New York: Routledge.

Latour, Bruno (2008): What is the Style of Matters of Concern? Two Lectures in Empirical Philosophy, Spinoza Lectures: University of Amsterdam, Publication of the Department of Philosophy (independent pamphlet).

Maasen, Sabine (2009): Wissenssoziologie, 2. komplett überarbeitete Aufl., Bielefeld: transcript.

Martin, Emily (1998): »Anthropology and the Cultural Study of Science«. Science, Technology \& Human Values 23(1), S. 24-43.

Martin, Emily (1997): »Anthropology and the Cultural Study of Science: From Citadels to String Figures«. In: James Ferguson/Akhil Gupta (Hg.), Anthropological Locations. Boundaries and Grounds of a Filed Science, Berkeley: University of California Press, S. 131-46.

Merton, Robert K. (1942/1973): »The Normative Structure of Science«.In: Norman W. Storer (Hg.), The Sociology of Science. Theoretical and Empirical Investigations, London: University of Chicago Press, S. 26778.

Merton, Robert K. (1938): »The Puritan Spur to Science«. In: Norman W. Storer (Hg.), The Sociology of Science. Theoretical and Empirical Investigations, London: University of Chicago Press S.228-53.

Merton, Robert K. (1957): Social Theory and Social Structure, Rev. and enl. ed. Glencoe Ill., New York: Free Press.

Merton, Robert K. (1965): On the Shoulders of Giants, a Shandean Postscript, New York: Free Press.

Merton, Robert K. (1938): »Science, Technology and Society in Seventeenth Century England«. In George Sarton (Hg.), OSIRIS: Studies on the History and Philosophy of Science and on the History of Learning and Culture. Brügge: The St. Catherine Press, 362-632 
Naroll, Raoul/Michik, Gary L./Naroll, Frada (1976): Human Relations Area Files inc. Worldwide Theory Testing, New Haven: Human Relations Area Files.

Nowotny, Helga/Scott, Peter/Gibbons, Michael (2003): ») Mode 2〈 Revisited: The New Production of Knowledge«. Minerva 41, S. 179-94.

Polanyi, Michael (1962): »The Republic of Science: Its Political and Economic Theory«. Minerva I(1), S. 54-73.

Rabinow, Paul/Marcus, George E./Faubion, James D./Rees, Tobias (2008): Designs for an Anthropology of the Contemporary, Durham/London: Duke University Press.

Rammert, Werner (1988): »Technisierung im Alltag. Theoriestücke für eine spezielle soziologische Perspektive«. In: Bernward Joerges (Hg.), Technik im Alltag, Frankfurt/M.: Suhrkamp, S. 165-97.

Ravetz, Jerome (1971): Scientific Knowledge and its Social Problems, Oxford: Clarendon Press.

Schelsky, Helmut (1965) »Der Mensch in der technischen Zivilisation«. In: Helmut Schelsky (Hg.), Auf der Suche nach der Wirklichkeit, Düsseldorf: Diederichs.

Simon, Dagmar/Knie, Andreas/Hornbostel, Stefan (2001): Handbuch Wissenschaftspolitik. Wiesbaden: VS Verlag.

Stapelfeld, Gerhard (2005): Zur deutschen Ideologie. Soziologische Theorie und gesellschaftliche Entwicklung in der Bundesrepublik, Münster: LIT Verlag.

Tiedemann, Rolf/ Adorno, Theodor W. (1990): Gesammelte Schriften. Teil 8: Soziologische Schriften. 3. Aufl., Frankfurt/M.: Suhrkamp.

Warneken, Bernd Jürgen (2006): Die Ethnographie popularer Kulturen Eine Einführung, Wien/Köln/Weimar: Böhlau-Verlag.

Weber, Max (1934): Die protestantische Ethik und der Geist des Kapitalismus, Tübingen: J.C.B. Mohr.

Weber, Max (1922/2002): »Wirtschaft und Gesellschaft - Grundriß der verstehenden Soziologie«. In: Johannes Winckelmann (Hg.), ebd., 5. Aufl., Tübingen: Mohr.

Weingart, Peter (2003): Wissenschaftssoziologie, Bielefeld: transcript. 